\title{
EVALUACIÓN DE IMPACTO DEL COMERCIO ELECTRÓNICO EN LAS VENTAS DE LAS PYMES EN COLOMBIA: UN ANÁLISIS ECONOMÉTRICO
}

\section{IMPACT EVALUATION OF ELECTRONIC COMMERCE ON THE SALES OF SMES IN COLOMBIA: AN ECONOMETRIC ANALYSIS}

\author{
Juan David Corrales-Liévano ${ }^{1}$, Cindy Johanna Ruiz-Medina ${ }^{2}$ y Milton Fernando Angulo-Camargo ${ }^{3}$ \\ ${ }^{1}$ Universidad Militar Nueva Granada, Bogotá D.C, Colombia.Email: juan.corrales@ unimilitar.edu.co \\ ${ }^{2}$ Departamento Administrativo Nacional de Estadística -DANE, Colombia.Email: cjruizm@dane.gov.co \\ ${ }^{3}$ Universidad Militar Nueva Granada, Bogotá D.C, Colombia. Email: milton.anguloc@unimilitar.edu.co
}

Para citar este artículo: Corrales-Liévano. J.D., Ruiz-Medina, C. J., y Angulo-Camargo, M. F. (2019). Evaluación de impacto del comercio electrónico en las ventas de las pymes en Colombia: un análisis econométrico. Clío América, 13(26), 328-339. doi: http://dx.doi.org/10.21676/23897848.3558

Recibido: 22 mayo de 2019

Aceptado: 08 de agosto de 2019

Publicado en línea: noviembre 28 de 2019

\section{Palabras clave: comercio electrónico; empresas; tecnologías; análisis econométrico; impacto.}

JEL: A90; A29

\section{RESUMEN}

El comercio electrónico (CE) se ha convertido en un eje primordial en las diferentes sociedades. La tecnología se ha constituido como parte fundamental en el entorno empresarial y en la forma como se desarrollan y conectan las diferentes organizaciones en el mundo. Hay un número creciente de compañías que generan sistemas alternativos para incrementar sus ventas utilizando las tecnologías de información y comunicación (TIC). De aquí que el presente estudio pretenda determinar la influencia del e-commerce en las ventas de las pequeñas y medianas empresas (pymes) colombianas. Este documento comienza analizando un panel de 2177 empresas, donde se analizan los sectores de industria, comercio y servicios entre los años 2012 a 2016. Se pretende conocer el efecto causal que tiene el comercio en línea frente a las ventas totales realizadas por las compañías, así como su impacto en las ventas desarrolladas por cada una de ellas. Mediante un análisis econométrico, aplicando el modelo de medición de impacto denominado "Diferencias en Diferencias", se encontró que la adopción del comercio electrónico en las empresas medianas tiene mayor impacto que en las empresas pequeñas y que la actividad en el que más influye el comercio electrónico es en el sector servicios.

\section{ABSTRACT}

Electronic commerce (EC) has become a primary axis in different societies. Technology has become a fundamental part of the business environment, and the way organizations in the world are developed and connected. There is a growing number of companies that are generating alternative systems to increase their sales using Information and Communication Technologies (ICT). Hence, this study aims to determine the influence of e-commerce on the sales of Colombian Small and Medium-sized enterprises (SMEs). This paper starts by testing a panel of 2.177 companies, where the industry, commerce, and service sectors are analyzed between 2012 and 2016. It is intended to know the causal effect of online trading compared to the total sales made by companies, as well as its impact on the sales developed by each of them. Through an econometric analysis, applying the Differences in Differences impact measurement model, it was found that the adoption of Electronic Commerce in medium-sized companies has a greater impact than in small companies, and that the activity that most influences e-commerce is in the service sector. 


\section{EVALUACIÓN DE IMPACTO DEL COMERCIO ELECTRÓNICO EN LAS VENTAS DE LAS PYMES EN COLOMBIA: UN ANÁLISIS ECONOMÉTRICO}

\section{INTRODUCCIÓN}

La globalización económica ha obligado a los países del mundo a crear estrategias de acercamiento entre las economías para facilitar las transacciones entre ellos y ser más productivos y competitivos, mientras se exploran y explotan nuevos mercados y formas de innovación de los procesos. Con esto se conseguiría optimizar los recursos, tener mejores utilidades y llegar a más clientes.

El aumento de transacciones digitales entre empresas y clientes en el país (Suárez , 2019) ha evidenciado la necesidad de implementar nuevas tecnologías. Dicho suceso ha propiciado que tanto empresas como consumidores adopten nuevos mecanismos que permitan el acceso rápido y eficiente a información, bienes y servicios. De aquí que el presente artículo pretenda mostrar el efecto que ha tenido la adopción del Comercio Electrónico (CE) sobre las ventas de las Pequeñas y Medianas Empresas (pymes) en Colombia para los años entre 2012 y 2016, a través de un análisis econométrico que aplica el método de evaluación de impacto "Diferencias en Diferencias", con información de las Encuestas de Comercio, Industria y Servicios realizadas por el Departamento Administrativo Nacional de Estadística (DANE).

Los resultados se presentan por los mismos sectores a los que se les hizo la encuesta y de acuerdo con el tamaño de las empresas: pequeñas y medianas. Consecuentemente, se diseñó un modelo de diferencias en diferencias para estimar el impacto de la implementación del CE sobre las ventas, y un modelo de regresión lineal para observar la significancia de los estimadores obtenidos. Los datos muestran la relación entre el CE y las ventas en las pymes colombianas.

\section{Tecnologías de Información y Comunicación (TIC)}

Las TIC en Colombia vienen desarrollando una visible expansión. En las últimas décadas han promovido cambios culturales, políticos y sociales, y se han reconocido como instrumentos para mejorar la calidad de vida de las personas. Esto porque ellas permiten disminuir la desigualdad en el acceso y la producción de conocimiento y dinamizan el desarrollo económico y social.

De acuerdo con la Comisión de Regulación de Comunicaciones en Colombia (CRC, 2010), el país cuenta con una cobertura del $100 \%$ en acceso a telecomunicaciones, tanto en redes fijas como móviles. Sin embargo, en materia de servicios hay un estancamiento en cuanto al uso de voz fija y móvil, y radio y televisión. Por el contrario, el uso de los servicios de acceso a internet son los que están apalancando el crecimiento del sector TIC en Colombia. La Comisión también sostiene que el usuario de las TIC en el país tiene un interés creciente en la adquisición de dichas tecnologías. Esto es soportado en el aumento del gasto en TIC y en servicios, como la conexión a las redes sociales y la mensajería electrónica.

De acuerdo con lo anterior, el crecimiento de la economía colombiana guarda estrecha relación con el crecimiento del sector de telecomunicaciones (CRC, 2012). Entre el primer trimestre de 2012 y de 2018 el sector muestra coincidencias con la economía nacional (figura 1).

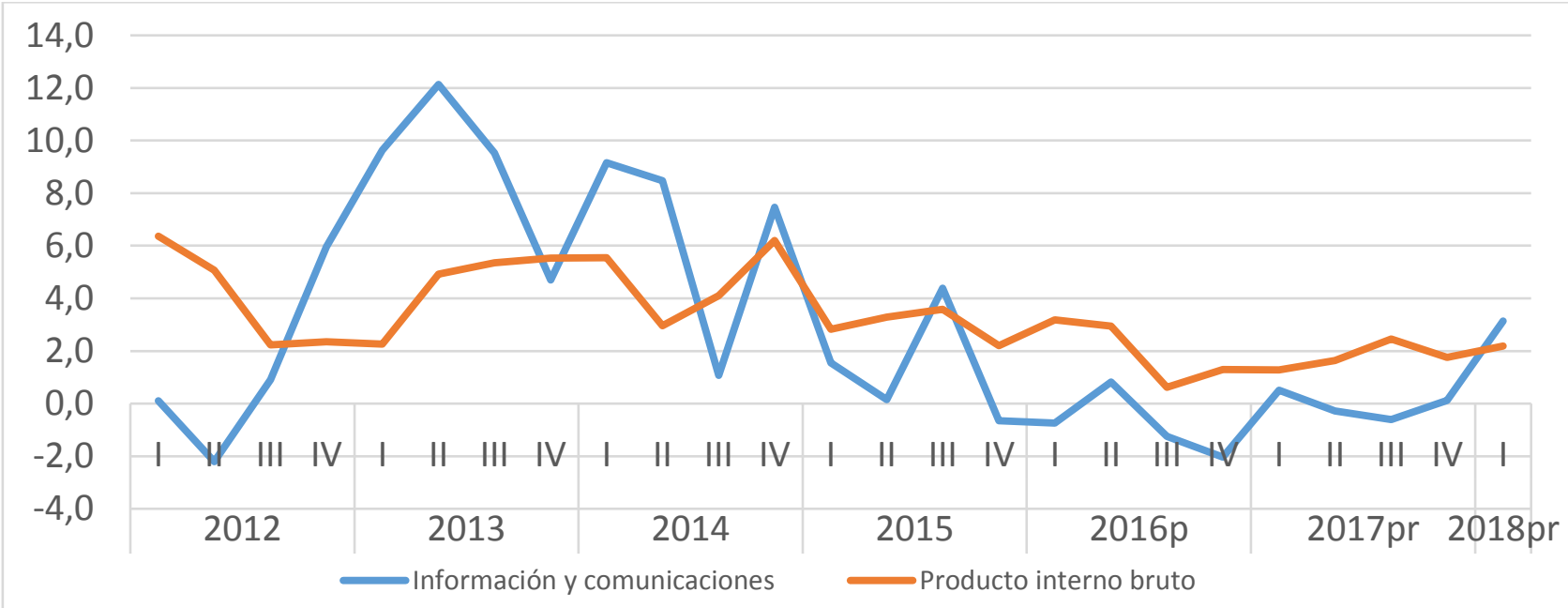

Figura 1. Crecimiento del PIB y del Sector Información y comunicaciones en Colombia (2012/I-2018/I).

Fuente: DANE, 2018. 
Aunque en Colombia se encuentran tiendas online de alto nivel en las principales categorías de CE (viajes y turismo, electrónica de consumo, computación y libros), aún hay una gran cantidad de productos que los consumidores adquieren desde tiendas online que operan fuera del país.

\section{Comercio Electrónico}

En los últimos años, el término $\mathrm{CE}$ se escucha frecuentemente debido a la expansión del internet y las demás tecnologías de la información, lo que está transformando la economía industrial del siglo pasado en economía digital. En esta última existen cada vez mejores modelos y enfoques tecnológicos para hacer negocios. Por lo tanto, las empresas han tenido la necesidad de adoptar el CE como estrategia para el desarrollo de sus procesos y relaciones comerciales, con el fin de mantenerse a la vanguardia a nivel tecnológico. En los países en desarrollo, el CE se encuentra en una tasa de adopción y uso bastante baja en comparación con las economías desarrolladas. Existen múltiples definiciones de CE (Espinoza Saucedo, Cano Anguiano y Lira Obando, 2016; Mazzarol, 2015;
Organización Mundial de Comercio; OMC, 2018; Comisión Europea; 2016; Mazzarol, 2015), aunque todas ellas tienen en común el uso de medios electrónicos para la compra y la venta de bienes o servicios. Es así como la digitalización ha contribuido en la disminución de grandes inversiones y desembolsos en infraestructura física para lograr presencia global, lo que ha llevado a una transformación en la forma de hacer negocios en todo el mundo (Alyoubi, 2015).

En Latinoamérica se destaca Brasil como el país con más usuarios de internet. Por un lado, la facturación en el año 2015 por este medio representó el 55,9 \% del total de América Latina (Neupavert, 2015). Eso deja en evidencia que existen países en la región que tienen una mayor adopción de CE que Colombia. Sin embargo, la brecha se está haciendo cada vez más pequeña, pues, en cuanto a ventas en línea de tecnología, tiquetes aéreos, boleterías entre otros, Colombia está a la vanguardia de este segmento del mercado (Pardo, 2008). La figura 2 muestra la tasa de crecimiento para América Latina en 2017 y 2018:

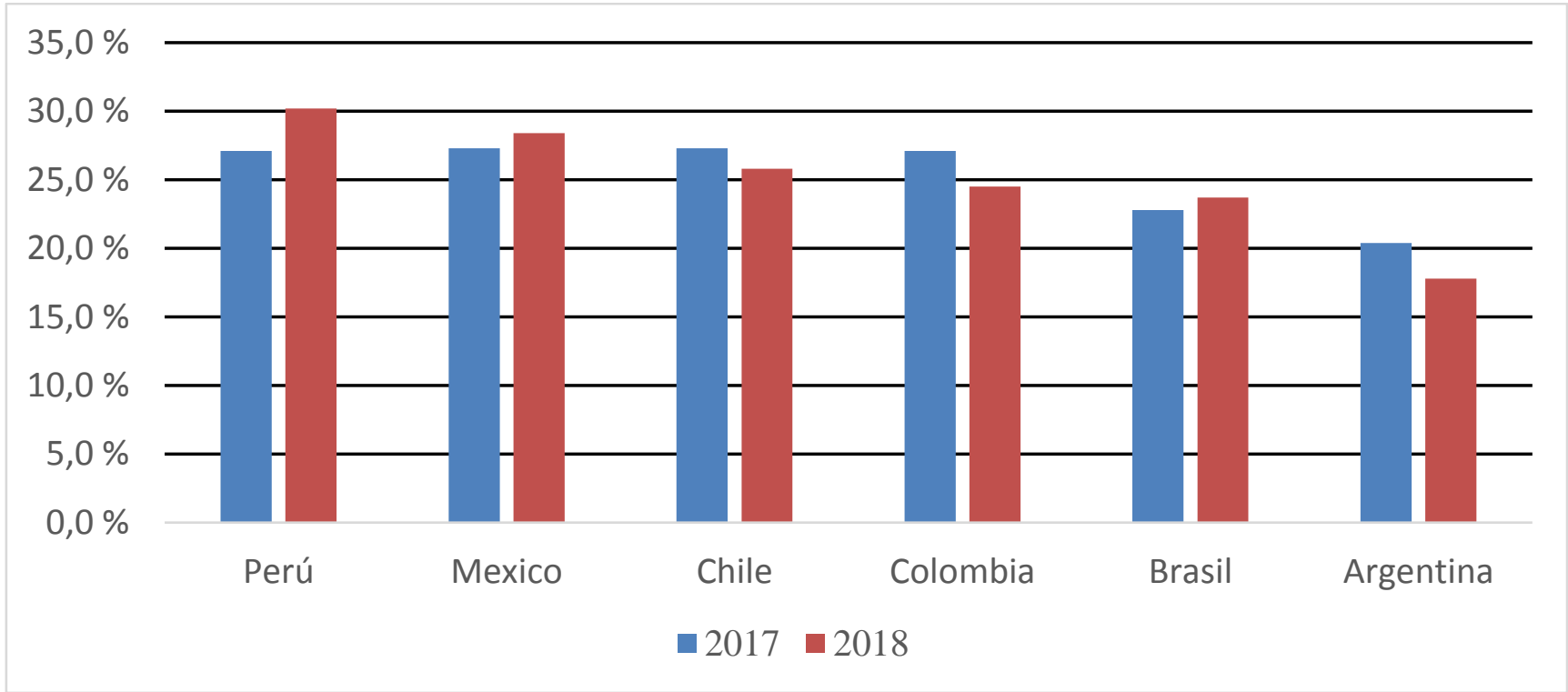

Figura 2. Tasa de crecimiento del CE en países seleccionados de América Latina en 2017 y 2018. Fuente: Statista (2018). Fuente: DANE, 2018.

Aunque en Colombia se encuentran tiendas online de alto nivel en las principales categorías de CE (viajes y turismo, electrónica de consumo, computación y libros), aún hay una gran cantidad de productos que los consumidores adquieren desde tiendas online que operan fuera del país.

\section{METODOLOGÍA}

Para lograr el objetivo de este trabajo, se recurrió a una investigación cuantitativa a nivel descriptivo y se establecieron los sectores económicos sobre los cuales se realizaría el análisis. De acuerdo con la información disponible en la sala de procesamiento especializada del DANE, fue elaborada la base de datos que consistió en un panel para registros de los años 2012 a 2016, con cifras de las pymes que reportan información a la Encuesta Anual de Comercio (EAC), la Encuesta Anual Manufacturera (EAM) y la Encuesta Anual de Servicios (EAS), llevadas a cabo por el DANE.

Para desarrollar un método de medición de impacto que 
fuera adecuado para los datos disponibles, se requirió de la revisión bibliográfica de modelos de medición aplicados en diversos ámbitos sociales, políticos y económicos. Esta revisión fue importante debido a que no es prudente aplicar métodos de medición sin fundamentos académicos. Se eligió entonces el método de diferencias en diferencias por ser el que mejor se adapta al análisis de impacto de los datos objeto del presente estudio. Con este modelo se pueden tener estimaciones confiables del efecto de la adopción del CE.

El método de diferencias en diferencias considera:

- La diferencia promedio de los resultados entre los individuos del grupo de tratamiento y el grupo de control.

- La diferencia entre la variable resultado (ventas), antes y después del tratamiento (adopción del $\mathrm{CE})$.

El principal objetivo de este artículo, como se dijo anteriormente, es analizar el impacto del CE en las ventas totales de las pymes colombianas. En concreto, la pregunta formulada es:

¿La adopción del CE tiene influencia en las ventas totales de las pymes?

Se plantean las siguientes hipótesis:

H1: La adopción del CE tiene impacto sobre las ventas totales de las empresas.

$\mathrm{H} 2$ : El sector económico en el que operan las empresas influye en el impacto del CE sobre las ventas.

H3: El tamaño de la empresa es un factor determinante para la adopción del CE.

La población de estudio puede identificarse en empresas colombianas que se encuentran en las Encuestas Anuales de Comercio, Industria y Servicios realizadas por el DANE. El análisis se hace para empresas pequeñas y medianas de cada sector comprendidas de la siguiente manera:

> Sector Comercio: Medianas 1226, pequeñas 2699.

> Sector Industria: Medianas 553, pequeñas 166.

$>$ Sector Servicios: Medianas 1421, pequeñas 756.

Las variables analizadas y su correspondiente descripción para el presente estudio se muestran en la Tabla 1:

Tabla 1. Variables utilizadas en el análisis econométrico

\begin{tabular}{|c|c|c|}
\hline Variable & Descripción & Categorías \\
\hline Empresa & Identificador de cada empresa & $\begin{array}{l}\text { Número asignado por el DANE a cada } \\
\text { empresa encuestada. }\end{array}$ \\
\hline Tipo & Tamaño de la empresa & $\begin{array}{l}\text { Pequeña } \\
\text { Mediana }\end{array}$ \\
\hline Sector & Sector económico de la empresa & Comercio, industria, servicios \\
\hline Venta & Ventas totales de la empresa & $\begin{array}{l}\text { Total de ventas realizadas por la empresa por } \\
\text { cada año }\end{array}$ \\
\hline Realiza_CE & Realiza o no ventas por CE & $\begin{array}{l}\text { 1-sí } \\
\text { 2-no }\end{array}$ \\
\hline Venta_CE & $\begin{array}{l}\text { Total de ventas realizadas por medio de } \\
\text { CE }\end{array}$ & $\begin{array}{l}\text { Cantidad vendida por medios electrónicos en } \\
\text { cada año }\end{array}$ \\
\hline Edad & $\begin{array}{l}\text { Corresponde a los años transcurridos } \\
\text { entre el año de constitución de la } \\
\text { empresa hasta } 2016\end{array}$ & Años en operación \\
\hline
\end{tabular}

Fuente: elaboración propia.

\section{Modelo}

El método diferencias en diferencias permite eliminar las características especiales de cada uno de los grupos (empresas sin CE y empresas que lo adoptaron). Además, admite la comparación intertemporal, dado que los efectos que se encuentran luego de adoptar el CE son comparables con los efectos antes de la adopción. Este método tiene un componente espacial, el cual se refiere a cada una de las observaciones (empresas), y un componente temporal (años) que hace referencia al comportamiento de las observaciones en los años que se comparan. 
Conocer el efecto causal del $\mathrm{CE}$ a nivel individual requiere definir un indicador de la adopción $D_{i}$, que es una variable binaria para cualquier empresa i-ésima que en este caso pueda o no adoptar el CE. Por lo tanto:

$D_{i}$

$=0$, indica que la empresa $i$ no adoptó comercio electrónico $D_{i}$

$=1$, indica que la empresa $i$ adoptó comercio electrónico

Para el objetivo de este trabajo se considera solamente una variable respuesta $Y_{i}$ que representa las ventas totales de la empresa $i$-ésima

Para cada empresa puede observarse la variable respuesta definida así:

$$
Y_{i}(t)=D_{i} * Y_{1 i}(t)+\left(1-D_{i}\right) * Y_{0 i}(t)
$$

\section{Control del sesgo}

La comparación de los valores de $Y(t)$, realizada únicamente para las empresas que adoptaron el CE, puede resultar sesgada antes y después de la adopción, debido a que es posible que se tengan en cuenta tendencias temporales sobre la variable respuesta (ventas) o porque durante el desarrollo del ejercicio aparecen otras variables que intervienen en el valor de las ventas y que no estaban presentes antes de la adopción. Para evitar este sesgo se recurre al grupo control que son las empresas que no adoptaron CE en ningún momento del periodo entre 2012 y 2016. Con este grupo se detectará la variación temporal de las ventas, ajena a la implementación del CE.

Entonces, se definen las siguientes variables:

$Y(t)$ : ventas observadas en el periodo $t$

$\mathrm{Y}_{1}(\mathrm{t})$ : ventas en el periodo $\mathrm{t}$ de las empresas con comercio electrónico $\mathrm{Y}_{0}(\mathrm{t})$ : ventas en el periodo $\mathrm{t}$ de las empresas sin comercio electrónico $t=0$ : antes de adoptar el comercio electrónico

$\mathrm{t}=1$ : despues de adoptar el comercio electrónico

Se elimina el subíndice $i$ debido a que no es posible observar los valores $Y_{1}(t)$ y $Y_{0}(t)$ para la misma empresa simultáneamente (Cansino y Sánchez, 2009, p.9). Por lo tanto, la ecuación 1 con la nueva notación es

$$
Y(t)=D * Y_{1}(t)+(1-D) * Y_{0}(t)
$$

El método de diferencias en diferencias supone que la tendencia temporal registrada por los individuos pertenecientes al grupo de control sirve a modo de variable "proxy", para conocer la evolución que hubieran seguido los beneficiarios del programa en el caso de que no hubiesen tomado el CE (Athey y Imbens, 2006).
Se construye el estimador $\alpha$ a partir de la diferencia entre las medias de las ventas de los dos grupos (tratamiento y control) antes de la adopción del CE, así como de la diferencia de medias de las ventas después de la adopción del CE. Luego se estima la "diferencia en diferencias".

$$
\begin{aligned}
& \alpha_{D I D}=\left\{\frac{1}{n_{1}} \sum_{\substack{i=1 \\
D=1}}^{n_{1}} Y_{i}(1)-\frac{1}{n_{0}} \sum_{\substack{i=1 \\
D=0}}^{n_{0}} Y_{i}(1)\right\} \\
& -\left\{\frac{1}{n_{1}} \sum_{\substack{i=1 \\
D=1}}^{n_{1}} Y_{i}(0)-\frac{1}{n_{0}} \sum_{\substack{i=1 \\
D=0}}^{n_{0}} Y_{i}(0)\right\}= \\
& =\frac{1}{n_{1}} \sum_{\substack{i=1 \\
D=1}}^{n_{1}}\left\{Y_{i}(1)-Y_{i}(0)\right\}-\frac{1}{n_{0}} \sum_{\substack{i=1 \\
D=0}}^{n_{0}}\left\{Y_{i}(1)-Y_{i}(0)\right\}=
\end{aligned}
$$

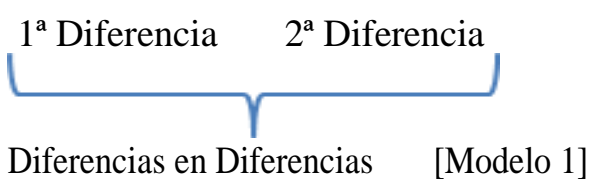

Este se puede estimar vía regresión a partir del modelo lineal:

$$
\begin{gathered}
Y_{i t}=\beta_{1}+\beta_{2} \operatorname{ConCE}+\beta_{3} \text { Después }+\delta\left(\text { ConCE }_{i} *\right. \\
\text { Después } \left._{t}\right)+e_{i t}
\end{gathered}
$$

Como se pretende evaluar el impacto de la adopción del CE en las ventas de las pymes por cada sector (comercio, industria y servicios), el método de diferencias en diferencias exige que en cada uno de ellos se tengan dos grupos de comparación:

Grupo tratamiento: pymes que no usaban el CE en 2012 y lo adoptaron en algún momento del año 2013.

Grupo control: pymes que no implementaron CE en ningún momento del periodo comprendido entre 2012 y 2016.

La muestra de los individuos del grupo tratamiento fue extraída de las empresas que adoptaron CE en el año 2013, para cada uno de los sectores comercio, industria y servicios. La muestra de los individuos del grupo control está conformada por las empresas que no adoptaron CE, para cada sector económico.

El inicio y el final del periodo determinan los momentos relevantes para el cálculo del estimador:

- Año 2013: se realizó la implementación del CE. 
- Años 2015 y 2016: se tiene el efecto de dicha adopción en las ventas.

Con el software STATA se procedió a modelar los cálculos de los coeficientes $\beta$ s de la ecuación (2) por medio de una regresión lineal para cada año, donde los valores de $Y_{1}(0)$ e $\mathrm{Y}_{0}(0)$ corresponden a las ventas de los individuos beneficiarios y de control, respectivamente, antes de que tenga lugar la adopción del CE. Así, se obtienen los valores de $Y_{1}(1)$ y de $Y_{0}(1)$ que corresponden a las ventas de los individuos beneficiarios y de control, respectivamente, en un periodo posterior a la adopción del CE.

$\beta=$ ventas de las empresas que adoptaron CE ventas de las empresas que no lo adoptaron

Al obtener los $\beta$ s se procede a realizar la diferencia en diferencias entre los años que se quieren comparar: 2013 y 2015/16. Así se obtienen todos los coeficientes del Modelo 1, planteado al comienzo.

Diferencias tratamiento - grupo control

$$
\begin{aligned}
& \alpha_{D I D}=\frac{1}{n_{1}} \sum_{\substack{i=1 \\
D=1}}^{n_{1}}\left\{Y_{i}(1)-Y_{i}(0)\right\} \\
&-\frac{1}{n_{0}} \sum_{\substack{i=1 \\
D=0}}^{n_{0}}\left\{Y_{i}(1)-Y_{i}(0)\right\}
\end{aligned}
$$

Por otra parte, se examina si los estimadores encontrados son significativos estadísticamente, pues este caso indicaría la ausencia de impacto. Para esto se realizan inferencias sobre ellos, con una regresión lineal expresada así:

$$
\begin{gathered}
\text { Venta }=\alpha_{0}+\alpha_{1} a \tilde{\mathrm{n}} o+\alpha_{2} r e a l i z a \_C E+\alpha_{3} a \tilde{\mathrm{n}} o \\
* \text { realiza_CE }
\end{gathered}
$$

Y se analiza el $\mathrm{P}>\mathrm{t}$ de la variable $a \tilde{n} o *$ realiza_CE, el cual deberá ser mayor al nivel de significancia para demostrar que el estimador es significativo estadísticamente. Así, se realiza la aceptación de que el estimador es diferente de cero con un nivel de significancia del $5 \%$.

\section{RESULTADOS}

Para medir el impacto de la adopción del CE en las ventas de las pymes, se presentan los resultados por sector económico y tamaño de las empresas, los cuales se analizaron a partir de las ventas totales de las compañías en cada sector.
Se entiende que para el grupo tratamiento se tomaron las empresas que en 2012 no tenían CE, con el fin de evitar los efectos anteriores a ese año en el análisis. La comparación se realizó entre las ventas del año 2013, cuando el grupo tratamiento adoptó el CE, y las ventas de los años 2015 y 2016. Esto porque con la aplicación del método se evidenció que el efecto de la adopción del CE no se manifestó en el año inmediatamente después, debido a que la implementación por parte de la empresa pudo haber ocurrido a finales del año 2013 y en el año 2014 no se observó su impacto.

\section{Sector Comercio}

El análisis mostrado en la Tabla 2 incluye 3925 empresas dedicadas a actividades de comercio. Se obtuvo un total de 1069 empresas con CE en 2016, notándose que cada año eran menos las empresas que lo implementan en este sector.

El estimador encontrado indicó que, para esta actividad económica, el incremento de las ventas en el año 2016 de las empresas que adoptaron el CE fue un $113 \%$ mayor que el incremento de las ventas de las empresas que no lo adoptaron entre el año 2012 y 2016. Para las empresas pequeñas se observaron resultados más favorables que en las empresas medianas. Aun así, al ser más las empresas pequeñas las que conforman este sector, implica que en general los resultados fueron favorables.

Para la empresa mediana se encontró que la adopción del CE no se vio representado favorablemente en el mediano plazo (dos o tres años después de la adopción) en las ventas. En este grupo fueron 74 empresas las que adoptaron CE, mientras que 674 empresas no lo implementaron en ningún año entre 2012 y 2016. Por lo tanto, aunque las ventas en general aumentaron para el año 2015 y 2016, tanto para el grupo tratamiento como para el grupo de control, el aumento en las ventas de las empresas medianas que no adoptaron CE fue mayor. A saber, para el año 2015 el incremento en las ventas promedio fue de $\$ 5290847 \mathrm{y}$ para el 2016 el incremento fue de $\$ 19409780$.

Para la empresa pequeña se identificó que la adopción del CE impactó las ventas totales, pues para el año 2015 y para 2016 se tuvieron estimadores positivos. Eso permitió concluir la existencia de un impacto favorable sobre las ventas promedio de las empresas que implementaron CE. Para el año 2015, el incremento de las ventas de las empresas que adoptaron el CE fue un $115 \%$ mayor que el incremento de las ventas de las empresas que no lo adoptaron.

Lo anterior permite visualizar que para el año 2016 el incremento de las ventas de las empresas que lo adoptaron 
Tabla 2. Resultados sector comercio

\begin{tabular}{|c|c|c|c|c|c|c|c|}
\hline \multicolumn{8}{|c|}{ Empresas Medianas } \\
\hline \multicolumn{4}{|c|}{$2013-2015$} & \multicolumn{4}{|c|}{$2013-2016$} \\
\hline \multicolumn{2}{|c|}{ Con $\mathrm{CE}$} & \multicolumn{2}{|c|}{ Sin CE } & \multicolumn{2}{|c|}{ Con CE } & \multicolumn{2}{|c|}{ Sin $\mathrm{CE}$} \\
\hline $\begin{array}{l}\text { Ventas } \\
\text { antes }\end{array}$ & 33829003 & $\begin{array}{l}\text { Ventas } \\
\text { antes }\end{array}$ & 28117476 & $\begin{array}{l}\text { Ventas } \\
\text { antes }\end{array}$ & 33829003 & $\begin{array}{l}\text { Ventas } \\
\text { antes }\end{array}$ & 28117476 \\
\hline $\begin{array}{c}\text { Ventas } \\
\text { después }\end{array}$ & 38802949 & $\begin{array}{c}\text { Ventas } \\
\text { después }\end{array}$ & 33408324 & $\begin{array}{c}\text { Ventas } \\
\text { después }\end{array}$ & 38528028 & $\begin{array}{c}\text { Ventas } \\
\text { después }\end{array}$ & 47527256 \\
\hline \multicolumn{4}{|c|}{ Diferencias } & \multicolumn{4}{|c|}{ Diferencias } \\
\hline Con CE & 4973946 & Sin CE & 5290847 & Con CE & 5699026 & Sin CE & 19409780 \\
\hline \multicolumn{2}{|c|}{$\begin{array}{c}\text { Diferencias en } \\
\text { Diferencias }\end{array}$} & \multicolumn{2}{|c|}{$\alpha=-316902$} & \multicolumn{2}{|c|}{$\begin{array}{c}\text { Diferencias en } \\
\text { Diferencias }\end{array}$} & \multicolumn{2}{|c|}{$\alpha=-13710754$} \\
\hline \multicolumn{8}{|c|}{ Empresas Pequeñas } \\
\hline \multicolumn{4}{|c|}{$2013-2015$} & \multicolumn{4}{|c|}{$2013-2016$} \\
\hline \multicolumn{2}{|c|}{ Con $\mathrm{CE}$} & \multicolumn{2}{|c|}{ Sin CE } & \multicolumn{2}{|c|}{ Con CE } & \multicolumn{2}{|c|}{ Sin CE } \\
\hline $\begin{array}{l}\text { Ventas } \\
\text { antes }\end{array}$ & 10036639 & $\begin{array}{l}\text { Ventas } \\
\text { antes }\end{array}$ & 10210366 & $\begin{array}{l}\text { Ventas } \\
\text { antes }\end{array}$ & 10036639 & $\begin{array}{l}\text { Ventas } \\
\text { antes }\end{array}$ & 10210366 \\
\hline $\begin{array}{l}\text { Ventas } \\
\text { después }\end{array}$ & 11583114 & $\begin{array}{l}\text { Ventas } \\
\text { después }\end{array}$ & 10930182 & $\begin{array}{l}\text { Ventas } \\
\text { después }\end{array}$ & 11722228 & $\begin{array}{l}\text { Ventas } \\
\text { después }\end{array}$ & 11430195 \\
\hline \multicolumn{4}{|c|}{ Diferencias } & \multicolumn{4}{|c|}{ Diferencias } \\
\hline Con CE & 1546476 & Sin $\mathrm{CE}$ & 719816 & Con CE & 1685589 & Sin $\mathrm{CE}$ & 1219829 \\
\hline \multicolumn{2}{|c|}{$\begin{array}{c}\text { Diferencias en } \\
\text { Diferencias }\end{array}$} & \multicolumn{2}{|c|}{$\alpha=826660$} & \multicolumn{2}{|c|}{$\begin{array}{c}\text { Diferencias en } \\
\text { Diferencias }\end{array}$} & \multicolumn{2}{|c|}{$\alpha=465760$} \\
\hline
\end{tabular}

* Los valores son dados en miles de pesos colombianos.

Fuente: elaboración propia basados en datos proporcionados por el DANE.

\section{Sector Industria}

En la Tabla 3 muestra que este es el sector donde menos empresas adoptaron el CE en el periodo analizado, pues de las 719 empresas que reportaron información en la Encuesta Anual Manufacturera, 397 no lo implementaron entre 2012 y 2016.

En las empresas medianas, para el año 2013 solamente el $10 \%$ implementó CE, por lo que, al comparar las ventas de los dos grupos, las ventas de las empresas sin CE representaron el $90 \%$ de las ventas totales realizadas por las empresas medianas. De modo que, para los dos periodos de comparación, se obtienen estimadores negativos que muestran la disminución en el promedio de ventas en las compañías que lo implementaron en el año 2013; mientras que para las empresas que no adoptaron en ningún año entre 2012 y 2016 el promedio de ventas aumentó en $\$ 5$ 037002 en 2015 y \$4 979370 en 2016.

En las empresas pequeñas, para el año 2015 se encontró que el incremento de las ventas en las empresas que adoptaron CE es $33 \%$ menor que el incremento de las ventas de las empresas que no lo implementaron.

Tabla 3. Resultados sector industria

\begin{tabular}{|c|c|c|c|c|c|c|c|}
\hline \multicolumn{7}{|c|}{ Empresas Medianas } \\
\hline \multicolumn{2}{|c|}{$2013-2015$} & \multicolumn{4}{c|}{$2013-2016$} \\
\hline \multicolumn{2}{|c|}{ Con CE } & \multicolumn{2}{c|}{ Sin CE } & \multicolumn{2}{c|}{ Con CE } & \multicolumn{2}{c|}{ Sin CE } \\
\hline Ventas antes & 729815153 & Ventas antes & 14593539 & Ventas antes & 729815153 & Ventas antes & 14593539 \\
\hline $\begin{array}{c}\text { Ventas } \\
\text { después }\end{array}$ & 717021048 & $\begin{array}{c}\text { Ventas } \\
\text { después }\end{array}$ & 19630541 & $\begin{array}{c}\text { Ventas } \\
\text { después }\end{array}$ & 690465668 & $\begin{array}{c}\text { Ventas } \\
\text { después }\end{array}$ & 19572909 \\
\hline
\end{tabular}


EVALUACIÓN DE IMPACTO DEL COMERCIO ELECTRÓNICO EN LAS VENTAS DE LAS PYMES EN COLOMBIA: UN ANÁLISIS ECONOMÉTRICO

\begin{tabular}{|c|c|c|c|c|c|c|c|}
\hline \multicolumn{4}{|c|}{ Diferencias } & \multicolumn{4}{|c|}{ Diferencias } \\
\hline Con CE & -12794105 & Sin CE & 5037002 & Con $\mathrm{CE}$ & -39349485 & Sin CE & 4979370 \\
\hline \multicolumn{2}{|c|}{$\begin{array}{c}\text { Diferencias en } \\
\text { Diferencias }\end{array}$} & \multicolumn{2}{|c|}{$\alpha=-17831107$} & \multicolumn{2}{|c|}{$\begin{array}{c}\text { Diferencias en } \\
\text { Diferencias }\end{array}$} & \multicolumn{2}{|c|}{$\alpha=-44328855$} \\
\hline \multicolumn{8}{|c|}{ Empresas Pequeñas } \\
\hline \multicolumn{4}{|c|}{$2013-2015$} & \multicolumn{4}{|c|}{$2013-2016$} \\
\hline \multicolumn{2}{|c|}{ Con CE } & \multicolumn{2}{|c|}{ Sin CE } & \multicolumn{2}{|c|}{ Con $\mathrm{CE}$} & \multicolumn{2}{|c|}{ Sin CE } \\
\hline Ventas antes & 4863041 & Ventas antes & 4327400 & Ventas antes & 4863041 & Ventas antes & 4327400 \\
\hline $\begin{array}{c}\text { Ventas } \\
\text { después }\end{array}$ & 5638715 & $\begin{array}{c}\text { Ventas } \\
\text { después } \\
\end{array}$ & 5496468 & $\begin{array}{c}\text { Ventas } \\
\text { después }\end{array}$ & 5560500 & $\begin{array}{c}\text { Ventas } \\
\text { después } \\
\end{array}$ & 6685489 \\
\hline \multicolumn{4}{|c|}{ Diferencias } & \multicolumn{4}{|c|}{ Diferencias } \\
\hline Con CE & 775674 & Sin $\mathrm{CE}$ & 1169068 & Con CE & 697459 & Sin $\mathrm{CE}$ & 2358089 \\
\hline \multicolumn{2}{|c|}{$\begin{array}{c}\text { Diferencias en } \\
\text { Diferencias }\end{array}$} & \multicolumn{2}{|c|}{$\alpha=-393394$} & \multicolumn{2}{|c|}{$\begin{array}{c}\text { Diferencias en } \\
\text { Diferencias }\end{array}$} & \multicolumn{2}{|c|}{$\alpha=-1660630$} \\
\hline
\end{tabular}

Fuente: elaboración propia basados en datos proporcionados por el DANE. Los valores son dados en miles de pesos colombianos.

\section{Sector Servicios}

En el análisis mostrado en la Tabla 4 se aplicó la metodología en 2248 empresas dedicadas a la prestación de servicios. Se encontró que el CE tiene impacto en las ventas de las empresas a mediano plazo.

Del mismo modo, es en las empresas medianas en donde la adopción del CE tiene mayor impacto sobre las ventas. Este resultado demuestra que en 2015 el incremento en las empresas que adoptaron CE en 2013 fue $13 \%$ mayor que el incremento en las ventas de las empresas sin CE. Es decir, que para las empresas medianas que lo adoptaron en 2013, el incremento en las ventas fue $62 \%$ mayor que el incremento de las ventas de las empresas que no lo adoptaron en todo el periodo 2012-2016.
Para la empresa pequeña, al hacer la estimación de diferencias en diferencias para las 756 empresas pequeñas para el año 2015, se observó que, tanto para las empresas que adoptaron CE en el año 2013 como para las que no lo implementaron en ningún año del periodo entre 2012 y 2016, las ventas promedio disminuyeron, de modo que no se puede medir el impacto de la adopción del CE en este año.

Por otro lado, se obtuvo que la diferencia entre el incremento en las ventas de las empresas con CE y las empresas que no lo implementaron en todo el periodo de 2012 a 2016 es de $\$ 939$ 902. Se observó que el promedio de ventas disminuyó para los dos grupos en el año 2016. Sin embargo, la disminución fue mayor en las ventas promedio de las empresas que no implementaron CE.

Tabla 4. Resultados sector servicios

\begin{tabular}{|c|c|c|c|c|c|c|c|}
\hline \multicolumn{8}{|c|}{ Empresas Medianas } \\
\hline \multicolumn{4}{|c|}{$2013-2015$} & \multicolumn{4}{|c|}{$2013-2016$} \\
\hline \multicolumn{2}{|c|}{ Con $\mathrm{CE}$} & \multicolumn{2}{|c|}{ Sin CE } & \multicolumn{2}{|c|}{ Con $\mathrm{CE}$} & \multicolumn{2}{|c|}{ Sin CE } \\
\hline Ventas antes & 8653541 & Ventas antes & 9005432 & Ventas antes & 8653541 & Ventas antes & 9005432 \\
\hline $\begin{array}{l}\text { Ventas } \\
\text { después }\end{array}$ & 10524792 & $\begin{array}{l}\text { Ventas } \\
\text { después }\end{array}$ & 10657526 & $\begin{array}{l}\text { Ventas } \\
\text { después }\end{array}$ & 11229982 & $\begin{array}{c}\text { Ventas } \\
\text { después }\end{array}$ & 10598661 \\
\hline \multicolumn{4}{|c|}{ Diferencias } & \multicolumn{4}{|c|}{ Diferencias } \\
\hline Con CE & 1871252 & Sin CE & 1652094 & Con CE & 2576442 & Sin CE & 1593229 \\
\hline \multicolumn{2}{|c|}{$\begin{array}{c}\text { Diferencias en } \\
\text { Diferencias }\end{array}$} & \multicolumn{2}{|c|}{$\alpha=219157$} & \multicolumn{2}{|c|}{$\begin{array}{c}\text { Diferencias en } \\
\text { Diferencias }\end{array}$} & \multicolumn{2}{|c|}{$\alpha=983213$} \\
\hline \multicolumn{8}{|c|}{ Empresas Pequeñas } \\
\hline \multicolumn{4}{|c|}{$2013-2015$} & \multicolumn{4}{|c|}{$2013-2016$} \\
\hline
\end{tabular}


Juan David Corrales-Liévano, Cindy Johanna Ruiz-Medina y Milton Fernando Angulo-Camargo

\begin{tabular}{|c|c|c|c|c|c|c|c|}
\hline \multicolumn{2}{|c|}{ Con CE } & \multicolumn{2}{|c|}{ Sin CE } & \multicolumn{2}{|c|}{ Con CE } & \multicolumn{2}{|c|}{ Sin CE } \\
\hline Ventas antes & 4867996 & Ventas antes & 7402521 & Ventas antes & 4867996 & Ventas antes & 7402521 \\
\hline $\begin{array}{l}\text { Ventas } \\
\text { después }\end{array}$ & 4548671 & $\begin{array}{l}\text { Ventas } \\
\text { después }\end{array}$ & 7282816 & $\begin{array}{l}\text { Ventas } \\
\text { después }\end{array}$ & 4463126 & $\begin{array}{l}\text { Ventas } \\
\text { después }\end{array}$ & 6057749 \\
\hline \multicolumn{4}{|c|}{ Diferencias } & \multicolumn{4}{|c|}{ Diferencias } \\
\hline Con CE & -319325 & Sin CE & -119705 & Con CE & -404870 & Sin CE & -1344772 \\
\hline \multicolumn{2}{|c|}{$\begin{array}{l}\text { Diferencias en } \\
\text { Diferencias }\end{array}$} & \multicolumn{2}{|c|}{$\alpha=-199620$} & \multicolumn{2}{|c|}{$\begin{array}{c}\text { Diferencias en } \\
\text { Diferencias }\end{array}$} & \multicolumn{2}{|c|}{$\alpha=939902$} \\
\hline
\end{tabular}

*Los valores son dados en miles de pesos colombianos.

Fuente: elaboración propia basada en datos proporcionados por el DANE.

\section{DISCUSIÓN}

El CE tiene un papel en el proceso de globalización y en el desarrollo de los medios modernos de comunicación, especialmente electrónicos. El CE cambia radicalmente las relaciones de poder y del mercado global (Slavko, 2017). Este cambio supone mejores beneficios a un costo menor para las organizaciones, lo que incide en el crecimiento económico y en el PIB de una nación (Johri, 2016).

Los resultados del presente estudio evidencian que el CE posee un efecto positivo a largo plazo en lo relacionado con las ventas de una empresa. Esto es acorde con lo propuesto por Cecere y Acatitla (2016), quienes sostienen que la adopción del CE es una forma de permanecer en el mercado. Sin embargo, los frutos de su implementación en la empresa colombiana son escasos en el corto plazo, toda vez que no se evidencian diferencias significativas entre las ventas desarrolladas por las firmas que lo adoptaron y aquellas que no han desarrollado dicha implementación. Esto se asimila a lo propuesto por Corrales-Liévano (2019), quien no encontró evidencia estadística asociada a la relación de las ventas entre las empresas que poseen y no poseen ventas en línea, al desarrollar su investigación en el análisis de los datos en un mismo año.

No obstante, la presente investigación acudió al análisis entre 2013 y 2016, y su efecto entre estos años, lo que permitió establecer su comportamiento y evidenciar, mediante el método econométrico diferencias en diferencias, la existencia de un efecto positivo en las ventas de las empresas que adoptaron este mecanismo como estrategia comercial. De acuerdo con ello, el impacto de la implementación del CE en las empresas colombianas se da en el mediano y largo plazo.

Por otro lado, el CE en las pymes ha tenido un desarrollo lento en Colombia. Esto se evidencia en concordancia con lo propuesto por Falk y Hagsten (2015), quienes sostienen que el porcentaje de empresas que realizan actividades de ventas en línea, después de comenzar desde un nivel bajo, crece lentamente en el tiempo. Sin embargo, las necesidades del mercado han obligado a estas empresas a evolucionar con respecto a la oferta de bienes por medios electrónicos, pues "el crecimiento en acceso a red que está experimentando nuestro país ha permitido reducir cada vez más los costos, permitiendo que los portafolios del mercado se diversifiquen" (Pardo, 2008, p. 20).

Por tanto, la pequeña, mediana y grande empresa deben tener presente la filosofía de gestión tradicional, mientras que los modelos de operación deberían ajustarse para garantizar la competitividad de las empresas en el desarrollo del CE (Chen, Yu, y Hsieh, 2013). No obstante, los resultados de este estudio apoyan lo afirmado por TingKun, Chun-Hung, y Wen-Cheng (2015), quienes sostienen que las organizaciones con mayor tamaño, mejores ratios en el número de aplicaciones electrónicas tecnológicas y mayor productividad tienen más posibilidades de adoptar el CE.

\section{CONCLUSION}

El objetivo de este trabajo fue comprobar si la adopción del CE posee algún impacto en las ventas de las pymes colombianas y establecer en qué sector económico este impacto es más significativo. Además, se quiso identificar si son las empresas medianas o las pequeñas las que están más dispuestas a implementar el CE para sus transacciones comerciales.

$\mathrm{Al}$ respecto, todos los estimadores son significativos estadísticamente, lo que implica que el efecto de la variable Realiza_CE, la cual toma valor de 1 si la empresa adopta CE y de 0 si no lo implementa, no es nulo. Eso permite constatar la hipótesis H1: La adopción del CE tiene impacto sobre las ventas totales de las empresas.

De acuerdo con revisión de literatura se comprobó que la inversión y el crecimiento de la infraestructura tecnológica es un factor determinante en la adopción del CE, en cuanto este facilita el acceso a medios, a información y contribuye 
a mejorar las transacciones electrónicas, tanto por el lado de las empresas como de los consumidores.

Las políticas y las regulaciones dirigidas a las TIC deben tener en cuenta la importancia de generar confianza en los usuarios para el uso de las redes informáticas, pues en Colombia la desconfianza y el desconocimiento sobre las transacciones en línea han hecho que el país se encuentre rezagado en uso de tecnologías con respecto a Europa y Estados Unidos.

En Colombia, la principal fuente de rentabilidad del CE son las transacciones empresa a empresa, pues, aunque en el país está creciendo la cultura de compras por internet, los colombianos prefieren realizar compras en línea a empresas extranjeras porque generan más confianza.

Por otro lado, los resultados del análisis econométrico realizado en este trabajo muestran que en Colombia es en el sector servicios donde la adopción del CE tiene un mayor impacto, más que en los sectores de industria y comercio, en el periodo analizado 2012-2016. Esto confirma la hipótesis H2: El sector económico en el que operan las empresas influye en el impacto del CE sobre las ventas.

Es en las empresas pequeñas donde se encontró que la adopción del CE tiene un impacto positivo en las ventas para el sector comercio, mientras que en las empresas medianas el CE tiene un impacto positivo en el sector servicios.

Para los sectores industria y comercio, el porcentaje de empresas que adoptaron CE en el año 2013 fue muy similar para las empresas medianas y pequeñas de cada sector. Es decir, la adopción depende más del sector donde se encuentre la empresa que del tamaño. Por lo tanto, se refuta la H3: El tamaño de la empresa es un factor determinante para la adopción del CE.

\section{Declaración sobre conflictos de interés}

Este artículo es producto de investigación derivado del proyecto INV-ECO 2584 "Efecto del comercio electrónico en las ventas de las pymes tradicionales en la ciudad de Bogotá D.C." financiado por la Vicerrectoría de Investigaciones de la Universidad Militar Nueva Granada en vigencia 2018. Sus resultados son independientes con respecto a la institución financiadora, y durante la ejecución del trabajo o la redacción del manuscrito no incidieron intereses o valores distintos a los que usualmente tiene la investigación.

\section{REFERENCIAS BIBLIOGRÁFICAS}

Alyoubi, A. A. (2015). E-commerce in Developing Countries and how to Develop them during the Introduction of Modern Systems. Procedia Computer Science, 65(Iccmit), 479-483. doi:10.1016/j.procs.2015.09.127

Athey, S. y Imbens, G. W. (2006). Identification and Inference in Nonlinear Difference-inDifferences Models. The Econometric Society, 74(2), 431-497.

Cansino, J. M. y Sánchez, A. (2009). Cálculo del estimador de diferencias en diferencias aplicado a la evaluación de programas públicos de formación: métodos alternativos para su obtención a partir de datos simulados. Documento de Trabajo, Universidad de Sevilla, $1-26$.

Cecere, A. y Acatitla, E. (2016). El comercio electrónico (e-commerce): una posible ventana de oportunidad para las firmas en los países en vías de desarrollo. Revista Internacional de Investigación y Docencia 1(1), 12-19.

Chen, L. C., Yu, T. J. y Hsieh, C. J. (2013). KeyGraph-based chance discovery for exploring the development of e-commerce topics. Scientometrics, 95(1), 257-275. doi:10.1007/s11192-012-0826-2

Comisión de Regulación de Comunicaciones. (2010). Análisis del sector TIC en Colombia: Evolución y Desafíos. Documento de Análisis Regulación de Infraestructura y Centro de Conocimiento de la Industria.

https://www.crcom.gov.co/recursos_user/Document os_CRC_2011/Actividades\%20_Regulatorias/ AgendaRegulatoria/2011/DocumentoAnalisisI ndustria.pdf 
Corrales, L. J. D. y Gil, H. R. J. (2017). Efecto Del Comercio Electrónico En Empresas Tradicionales Del Sector Comercial En Colombia. Revista Global de Negocios, 6(2), $41-50$

Corrales, L. J. D. (2019). Examining the Relationship of Electronic Commerce in the Total Sales of Colombian Commercial SMEs: a Look Between the Years 2012 and 2016 in a Developing Country in South America. Revista Logos, Ciencia \& Tecnología, 11(3), 30-42. doi: 10.22335/rlct.v11i3.904

Departamento Administrativo Nacional de Estadisticas - DANE. (2018). Producto Interno bruto (PIB) (Tasa de crecimiento anual) https://www.dane.gov.co/files/investigaciones/ boletines/pib/Anexos_produccion_constantes_ I_2018.xlsx

Espinoza, S. L. E., Cano, A. R. G. y Lira, O. A. (2016). RECI Revista Iberomericana de las ciencias computacionales e informática. http://www.reci.org.mx/index.php/reci/article/ view/41/182

Falk, M. y Hagsten, E. (2015). E-commerce trends and impacts across Europe. International Journal of Production Economics, 170, 357369. doi:10.1016/j.ijpe.2015.10.003

Gutiérrez , E. (2015). Impacto del comercio electrónico en las grandes y medianas empresas de la ciudad de Sogamoso Boyacá, Colombia. Cuadernos Latinoamericanos de Administración, XI(20), 83-96. doi:10.18270/cuaderlam.v11i20.630

Johri, B. (2016). Econometric Analysis of the Relationship between Indian E-Commerce
Transaction and GDP. Productivity, 56(4), 319325.

http://search.proquest.com/docview/17799471 14? accountid $=45375$

Mazzarol, T. (2015). SMEs engagement with ecommerce, e-business and e-marketing. Small Enterprise Research, 22(1), 79-90.

Neupavert, M. (2015). El mercado del comercio electrónico en Brasil. Junio de 2015. Recuperado de https://www.icex.es/icex/GetDocumento?dDo cName $=$ DOC $2015496115 \&$ urlNoAcceso $=/$ ice $\mathrm{x} / \mathrm{es} /$ registro/iniciarsesion/index.html?urlDestino=https://www.ice x.es:443/icex/es/navegacion-principal/todosnuestros-servicios/informacion-demercados/paises/navegacion-princip

Observatorio de Ecommerce. (2018). Evolución y retos del comercio digital en Colombia. https://www.observatorioecommerce.com.co/e volucion-del-comercio-digital-en-colombia Organización Mundial de Comercio - OMC. (2018). Comercio electrónico. https://www.wto.org/spanish/tratop_s/ecom_s/ ecom_s.htm

Pardo, C. O. (2008). El Comercio Electrónico a Nivel Internacional $y$ en Colombia. http://biblioteca.unitecnologica.edu.co/notas/te sis/0063208.pdf

Slavko, Đ. (2017). Electronic Commerce. Economics, 4(2), 133-141. doi:10.1515/eoik2017-0003

Statista. (2018). Growth rate of e-commerce sales in selected Latin American countries in 2017 and 2018. 
EVALUACIÓN DE IMPACTO DEL COMERCIO ELECTRÓNICO EN LAS VENTAS DE LAS PYMES EN COLOMBIA: UN ANÁLISIS ECONOMÉTRICO

https://www.statista.com/statistics/880469/onli ne-shopping-growth-rate-latin-america/

Suárez, V. (2019). Compras y ventas en internet se aceleran.

https://www.elcolombiano.com/negocios/inno vacion/compras-y-ventas-en-linea-crecen-a-
paso-de-gigante-EB10334541

Ting-Kun, L., Chun-Hung, C. y Wen-Cheng, L. (2015). The Impact of E-Commerce Adoption on Operating Cost: An Empirical Study of Taiwanese Manufacturing Firms. International Journal of Economic Perspectives, 9(2), 71-84. 